\title{
CULTASK, the Coldest Axion Experiment at CAPP/IBS in Korea
}

\section{Woohyun Chung*t}

Center for Axion and Precision Physics Research, IBS, Korea

E-mail: gnuhcweibs.re.kr

\begin{abstract}
The axion is an excellent dark matter candidate motivated by the Peccei-Quinn solution to the strong-CP problem. The IBS Center for Axion and Precision Physics Research (CAPP) in Korea will explore the dark matter axion using a method suggested by P. Sikivie, converting axions into microwave photons in a resonant cavity permeated by a strong magnetic field. CAPP's first microwave axion experiment, CULTASK (CAPP's Ultra Low Temperature Axion Search in Korea) is being launched at KAIST (Korea Advanced Institute of Science and Technology) campus this spring utilizing top of the line equipment and technology. I will outline many R\&D efforts that are currently being undertaken to make the axion search range broader and the sensitivity greater. I will also discuss the results from the recent engineering runs and future plans of the axion experiment.
\end{abstract}

38th International Conference on High Energy Physics 3-10 August 2016

Chicago, USA

\footnotetext{
* Speaker.

${ }^{\dagger}$ A footnote may follow.
} 


\section{Axion research at CAPP}

The main research focus of the Center for Axion and Precision Physics Research (CAPP) at IBS is to establish a state-of-the-art axion experiment in Korea. The concept of CAPP's axion experiment is based on P. Sikivie's proposed method[1], searching for microwave axions in a high $\mathrm{Q}$ value cavity submerged in very high magnetic field. The scheme has been employed by a handful of axion experiments so far. The design concept of our experiment is similar to ADMX[2] (Axion Dark Matter eXperiment), but the mass range we are searching for the axion is about 10 times broader and we are also planning of make a number of improvements over ADMX.

CAPP's axion search experiment was named, CULTASK (CAPP Ultra Low Temperature Axion Search in Korea) since it is considered to be "the coldest axion experiment". The dilution refrigerator (BlueFors LD400 series) could provide ultra low temperature $(<10 \mathrm{mK}$ base temperature) to the cavity. No other axion experiment was ever done in this low temperature before. R\&D projects CAPP is persuing include ultra high field magnets wih large bore, SQUID or JPA amplifiers and high Q-factor cavity with superconducting coating.

The initial stage of building our axion experiment is completed at KAIST (Korea Advanced Institute for Science and Technology) Munji Campus with successful installation of two new dilution refrigerators (one with $8 \mathrm{~T}$ superconducting magnet) that could lower the temperature of cavities to less than $50 \mathrm{mK}$. A resonant cavity $(10 \mathrm{~cm} \mathrm{OD)}$ and the support structure were fabricated and installed with the frequency tuning system employing a sapphire rod driven by a piezoelectric actuator. RF receiver chain, including low noise HEMT amplifiers is set up and tested successfully with DAQ system. With R\&D projects in progress to improve sensitivity, we should be able to start taking physics quality data in early 2017.

\section{CAPP's R\&D Projects}

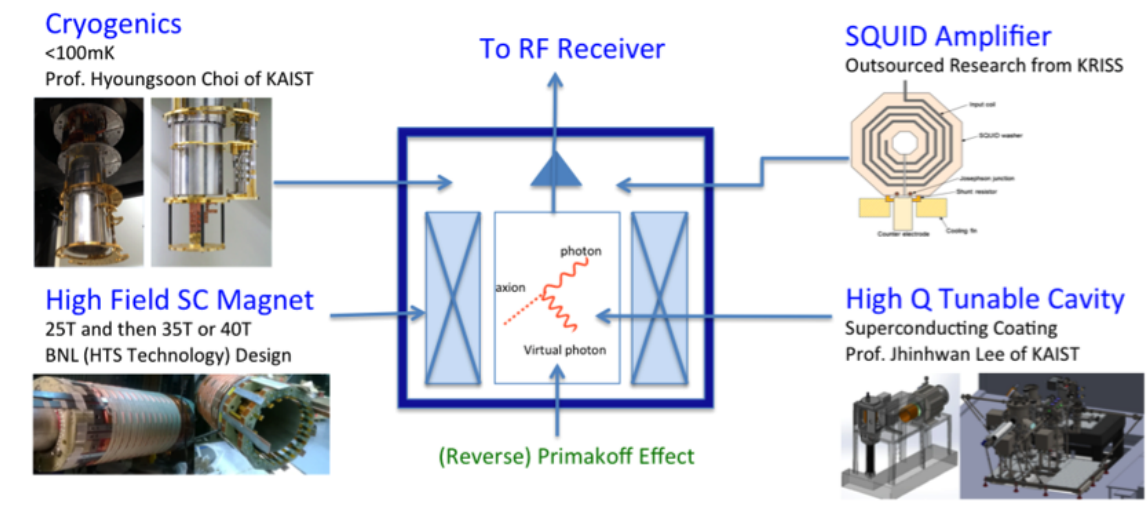

Figure 1: CAPP's R\&D projects for axion search

CAPP is driving a research and development plan to achieve the sensitivity required for the entire range of axion models. The R\&D plan (Figure 1) includes ultra high field superconducting magnets with various bore sizes (5, 10 and $35 \mathrm{~cm}$ innner diameter), high Q-factor superconducting 
cavities, high-gain gigahertz superconducting quantum interference device (SQUID) amplifiers and multi-cavity phase locking scheme for higher axion mass.

A powerful $25 \mathrm{~T}$ superconducting magnet will be developed and delivered by BNL(Brookhaven National Laboratory) in 2 to 3 years. This ultra high field magnet developed by BNL has exceptionally big $10 \mathrm{~cm}$ bore and is based on a next generation technology called HTS (High Temperature Superconductor). The compact (outer diameter of only $30 \mathrm{~cm}$ ) design of the magnet is intended to produce even higher field of 35T or 40T by adding another layer of LTS magnet outside in the future. If successful, this magnet will be the highest field superconducting magnet in the world with HTS technology.

Prof. Jhinhwan Lee of KAIST(Korea Advanced Institute of Science and Technology) is leading the effort of coating superconductors inside resonant cavities. Various configurations and different coating materials are being experimented now to overcome the effect of high magnetic field $(>8 \mathrm{~T})$ that goes through the cavity and to achieve the high Q-factor $\left(>10^{6}\right)$ for the resonant mode of choice $\left(T M_{010}\right)$.

The research and development of quantum limited SQUID amplifiers has been outsourced to KRISS (Korea Research Institute of Standards and Science), originally as a five-year project. The first sample of dc SQUID amplifiers (center frequency of $2.5 \mathrm{GHz}$ ) developed by Dr. Yong-Ho Lee of will be available for test before the end of 2016. We are also ordering commercial dc SQUID through a small company that had an experience of making similar amplifiers for ADMX.

\section{CAPP Ultra Low Temperature Axion Search in Korea (CULTASK)}

\begin{tabular}{|c|c|c|}
\hline & BF3 & BF4 \\
\hline Model & BlueFors LD400 & BlueFors LD400 \\
\hline Magnet & None & $8 \mathrm{~T}$ (AMI), $12 \mathrm{~cm}$ ID \\
\hline RF lines & 24 & 8 \\
\hline DC lines & 72 & 72 \\
\hline $\begin{array}{l}\text { Cool down } \\
\text { to }<10 \mathrm{mK}\end{array}$ & $20 \sim 24$ hours & $40 \sim 48$ hours \\
\hline $\begin{array}{l}\text { Base temp } \\
\text { at } \mathrm{MXC}\end{array}$ & $9 \mathrm{mK}$ & $\begin{array}{c}7 \mathrm{mK} \\
\text { w/ SC magnet }\end{array}$ \\
\hline $\begin{array}{l}\text { MXC temp } \\
\text { w/ Load }\end{array}$ & $\begin{array}{l}11 \mathrm{mk} w / \text { Al cavity }(4 \mathrm{~cm} \text { id) } \\
\text { and HEMT amp }\end{array}$ & $\begin{array}{l}30 \mathrm{mk} \text { w/ } 10 \mathrm{~kg} \text { OFHC copper } \\
\text { support structure and cavity } \\
\text { + HEMT amp } \\
\text { + Network Analyzer } \\
\text { + Piezo Controller }\end{array}$ \\
\hline
\end{tabular}

Figure 2: Properties of BlueFors LD400 Dilution Refrigerators at KAIST Munji campus

Two dilution refrigerators (BlueFors LD400) were delivered, installed and tested in March this year at CAPP's new laboratory located at KAIST Munji campus. One refrigerator(BF3) is designed for testing RF components (directional couplers, cryogenic circulators, resonant cavities and HEMT or SQUID amplifiers) and the other(BF4) refrigerator equipped with 8T superconducting magnet ( $12 \mathrm{~cm}$ inner bore) is for setting up a small scale axion engineering run. Figure 2 shows 
the characteristics of two new BlueFors dilution refrigerators. These refrigerators are designed to cool down and warm up relatively quick ( $20 \sim 24$ hours or $40 \sim 48$ hours when there is a $50 \mathrm{~kg}$ magnet attached) and fully automatic with a script so that it could cool down all the way with a touch of a button.

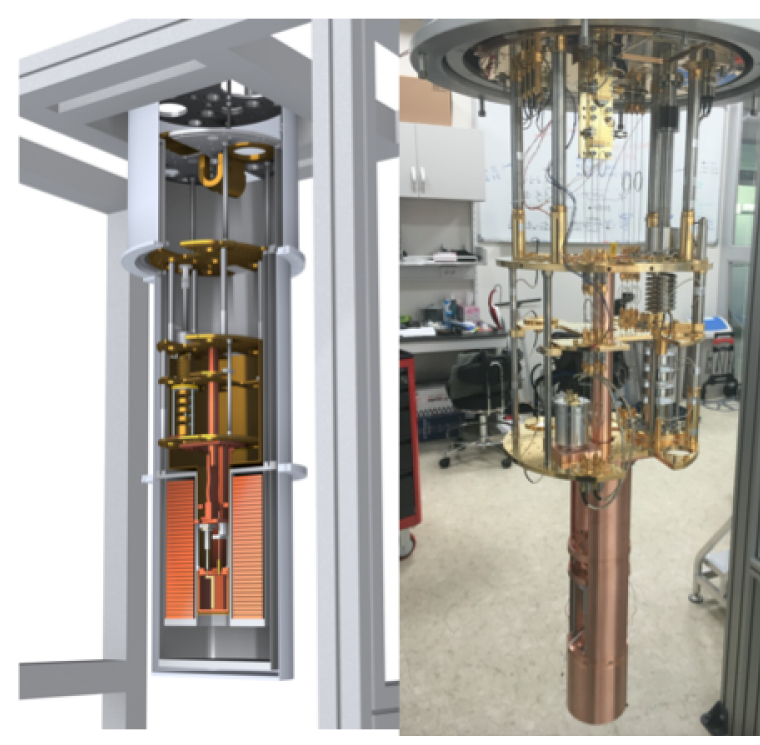

Figure 3: BlueFors LD400 dilution refrigerator with cavity and frequency tuning system installed (BF4)

We have designed a pure copper resonant cavity with $9 \mathrm{~cm}$ inner diameter $(2.5$ $\mathrm{GHz}$ for $T M_{010}$ mode) and a support structure with frequency tuning system (Figure 3). The physical temperature of the mixing plate which is thermally linked to the cavity and the support structure is around 30 $\mathrm{mK}$ now (7mK without cavity) with antenna probe and frequency tuning rod connected to piezoelectric actuators. Sapphire (dielectric) tuning rod is designed to be thermally linked to $100 \mathrm{mK}$ plate. The piezoelectric actuators are used to control frequency tuning rod (rotator) and probing antenna (linear) inside the refrigerator.

\subsection{High Q-factor cavity development}

Variety of sample cavities (OFHC Cu, $5 \mathrm{~N} \mathrm{Al}$ and $6 \mathrm{~N} \mathrm{Al}$ ) were prepared to test the enhancement of Q-factor in a cryogenic temperature. The technique of annealing is crucial for improving a Q-factor and we are at the stage of calibrating annealing furnace for now. The other important factor that has an impact on Q-factor is surface roughness. The high precision diamond-cutting technology is employed and the surface tolerance is less than $50 \mathrm{~nm}$.

\subsection{Superconducting Al cavity}

$\mathrm{Al}$ cavity becomes superconducting around $1.2 \mathrm{~K}$, which may not be very helpful for axion experiment with powerful magnetic field, because the critical field for $\mathrm{Al}$ is around $10 \mathrm{mT}$. However, it could provide us insights on how to design the cavity when superconducting coating is available. Al cavity of conventional design (cylinder with two lids) gets superconducting and Qfactor rises around 200,000 (TM $M_{010}$ mode) when the temperature is about $800 \mathrm{mK}$. Poor contact between lids and cylinder wall is suspected for not completely superconducting. The examination of $T E_{011}$ mode (no electric field along z direction) shows 2 to 20 million Q-factor (depending on the temperature). It is clear that the contact problem could be a limiting factor even when we have a superconducting cavity. One important lesson we learn from the simulation of cavity is that the Q-factor of $T M_{010}$ doesn't change when a perfect cavity is sliced vertically, even when split pieces were separated a little. We fabricated $\mathrm{Al}$ split cavity by machining two equal whole half pieces (by digging out half cylinder), effectively eliminating a contact problem, and the Q-factor goes up to 
over 2 million. It is also much easier to coat two half pieces (or many pieces) of cavity and we don't have to worry about the contact problem any more. We are actually applying this technique when coating superconductors to inner surface of cavities now.

\subsection{Engineering Run in 2016}

We are setting up a small scale, mid-to-low sensitivity axion experiment with BF4 as a starter. This detector includes $8 \mathrm{~T}$ superconducting magnet, $9 \mathrm{~cm}$ inner diameter OFHC Cu cavity, cryogenic RF chain (antenna probe, directional coupler, cryogenic circulators and amplifiers), and a system of room temperature RF receiver. In addition, there should be a DAQ system (CULDAQ) that controls, monitors the whole detector, and collects and stores data for analysis. The frequency tuning system with a sapphire tuning rod controlled by piezoelectric actuator is going through a precision test now and could be added later when we are confident.

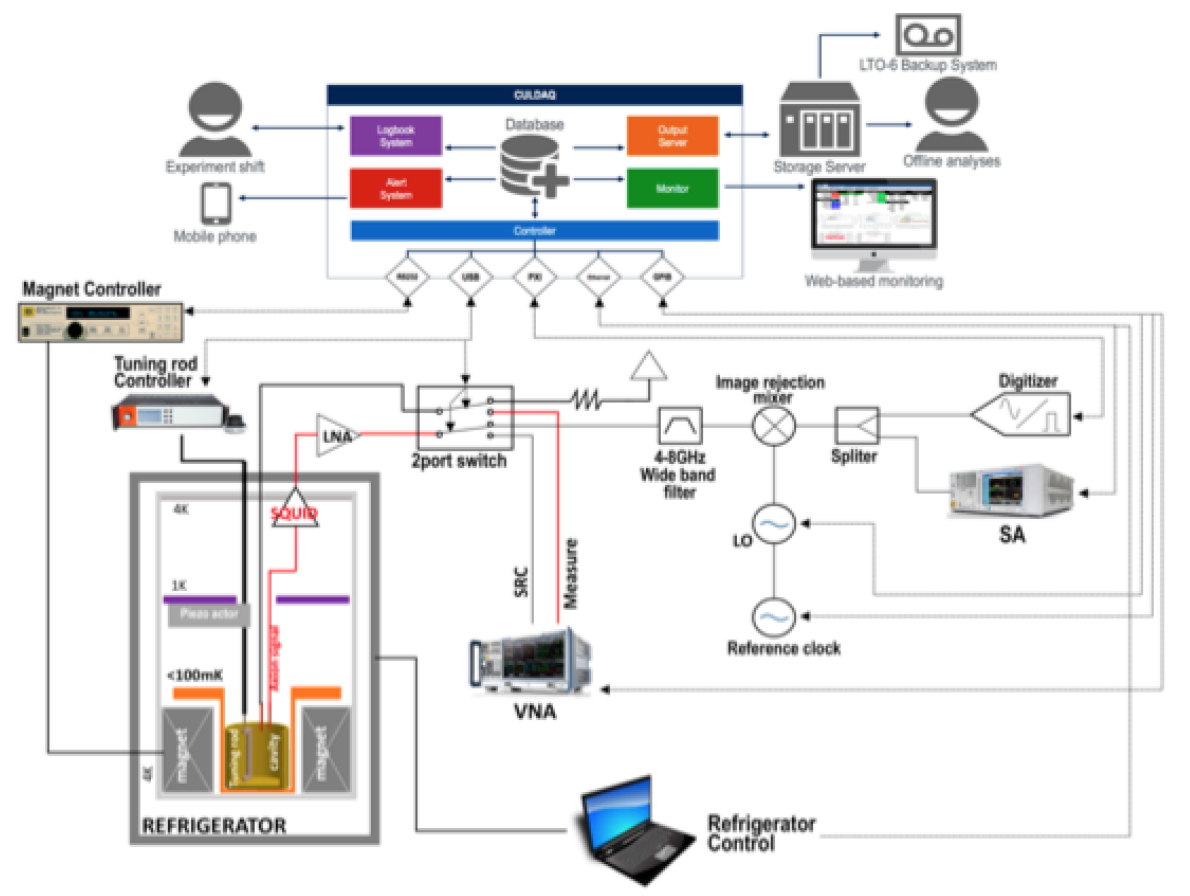

Figure 4: CULTASK DAQ system: CULDAQ

Figure 4 shows a complete axion detector set-up. We won't be able to reach KSVZ[3][4] sensitivity without SQUID amplifiers and superconducting cavity that should be available in a couple of years. However, this engineering run could provide us a great opportunity to prepare ourselves for evaluating and maximizing performance of other RF electronics components.

Cryogenic circulators (isolators) and amplifiers (HEMT) have been received, tested at KRISS by Dr. Yonuk Chong and will be verified at our BF3 refrigerator. The design and fabrication of room temperature RF receiver electronics setup is complete with mixers, filters and a spectrum analyzer. The digitization of signal and the recording of the data has been tested already. The data acquisition system (CULDAQ) that also controls and monitors switches, network analyzer, piezo controller, spectrum analyzer will be ready by September. 


\section{Plans beyond 2016}

The construction of 7 low vibration pads at the axion experimental site in KAIST Munji campus will be compete by the end of September and 4 more dilution refrigerators are to be installed early next year. Two 8T NbTi superconducting magnets (12 cm and $16 \mathrm{~cm}$ inner bore) will be used with two BlueFors dilution refrigerators and $18 \mathrm{~T} 5 \mathrm{~cm}$ bore HTS magnet is scheduled to be delivered in 2017. More superconducting magnets with much bigger bore sizes $(35 \mathrm{~cm}$ and $50 \mathrm{~cm})$ are also planned in the pipeline. Powerful magnets with different bore sizes along with the efforts on multi-cavity phase locking study would help us to search axions throughout the whole possible mass range. The improvements on booting axion conversion power by developing superconducting high-Q factor cavity and employing quantum limited SQUID amplifiers will pave a way to reach KSVZ QCD axion sensitivity region and beyond.

\section{Conclusion}

The preparation for the engineering run of CULTASK is complete at this stage and ready to go foward and collect physics data at least for a single frequency this year. The experimental space of 7 low vibration pads at KAIST Munji campus will be ready in a couple of months and more refrigerators are scheduled to be installed early next year (2017). The addition of SQUID amplifiers, ultra high field magnets and high Q-factor cavities with superconducting coating will improve the sensitivity and make a major contribution to axion research in coming years.

\section{Acknowledgments}

This work was supported by IBS-R017-D1-2016-a00/ IBS-R017-Y1-2016-a00.

\section{References}

[1] P. Sikivie, Phys. Rev. Lett. 41 (1983) 1415

[2] S. J. Asztalos et al., Nucl. Instrum. Methods Phys. Res., Sect. A 656, 39 (2011).

[3] J. E. Kim, Phys. Rev. Lett. 43, 103 (1979).

[4] M. A. Shifman, A. I. Vainshtein and V. I. Zakharov, Nucl. Phys. B 166, 493 (1980). 